\title{
BMJ Open Barriers and facilitators to physical activity in people with hip or knee osteoarthritis: protocol for a systematic review of qualitative evidence
}

\author{
Archontissa M Kanavaki, ${ }^{1}$ Alison Rushton, ${ }^{1}$ Rainer Klocke, ${ }^{2}$ Abhishek Abhishek, ${ }^{3}$ \\ Joan L Duda ${ }^{1}$
}

To cite: Kanavaki AM, Rushton A, Klocke R, et al. Barriers and facilitators to physical activity in people with hip or knee osteoarthritis: protocol for a systematic review of qualitative evidence. $B M J$ Open 2016;6:e012049. doi:10.1136/bmjopen-2016012049

- Prepublication history and additional material is available. To view please visit the journal (http://dx.doi.org/ 10.1136/bmjopen-2016012049).

Received 4 April 2016 Revised 15 September 2016 Accepted 13 October 2016

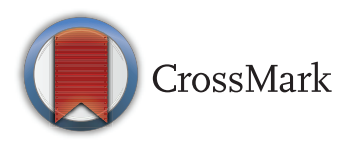

${ }^{1}$ School of Sport, Exercise and Rehabilitation Sciences, College of Life and Environmental Sciences, University of Birmingham, Birmingham, UK 2Department of

Rheumatology, Dudley Group NHS Foundation Trust, Dudley, UK

${ }^{3}$ Faculty of Medicine and Health Sciences, Academic Rheumatology Unit, School of Medicine, University of Nottingham, Nottingham, UK

Correspondence to Archontissa M Kanavaki; amk377@bham.ac.uk

\section{ABSTRACT}

Introduction: This protocol aims to describe the objective and methods to be followed in a systematic review of qualitative studies on barriers and facilitators to physical activity (PA) in people with hip or knee osteoarthritis (OA).

Methods and analysis: MEDLINE, EMBASE, PhychINFO, Web of Science, CINAHL, SPORTDiscus, Scopus and grey literature sources will be electronically searched. Hand search of qualitative research-centred journals, reference screening of relevant reviews and inquiries to researchers active in the field will complement the search. Studies will be selected if they apply qualitative or mixed-methods designs to directly explore factors that correspond to engagement in PA/ exercise or, the perceptions regarding PA/exercise in people with hip or knee OA. The Critical Appraisal Skills Programme Qualitative Checklist and the evaluative criteria of credibility, transferability, dependability and confirmability will be applied for the study appraisal. 2 independent reviewers will perform the search, study selection and study appraisal.

Thematic synthesis will be used for synthesising the findings of the primary studies and the process and product of the synthesis will be checked by a second researcher. ConQual approach will be used for assessing the confidence in the qualitative findings.

Ethics and dissemination: This systematic review will inform our understanding of the PA determinants and how to optimise behaviour change in people living with hip or knee $\mathrm{OA}$. The review findings will be reported in a peer-reviewed journal and presented at national or international conferences. The study raises no ethical issues.

Trial registration number: CRD42016030024.

\section{INTRODUCTION}

\section{Rationale}

Osteoarthritis (OA) is a common joint disease and one of the main causes of disability in ageing populations. ${ }^{1}$ Physical activity (PA) has a key role in the

\section{Strengths and limitations of this study}

- To the best of our knowledge, this is the first systematic review of qualitative evidence on barriers and facilitators of physical activity (PA) in people with hip or knee osteoarthritis (OA). Further, differences in barriers and facilitators between (1) exercise and lifestyle PA, and (2) uptake and maintenance of PA will be explored. This will largely contribute to our understanding of PA behaviours and provide information on how to optimise behaviour change in the population of interest.

- Rigorous methods will be applied informed by the Centre for Reviews and Dissemination and Cochrane Qualitative Research Methods Group guidelines and reported at all stages in line with the Enhancing Transparency in Reporting the Synthesis of Qualitative Research (ENTREQ) statement.

- The level of confidence in each review finding will be reported.

- One limitation of this systematic review is that only papers written in English will be included.

management of OA. For instance, exercise, which is a structured and purposeful form of $\mathrm{PA}^{2}$ is effective in reducing pain and improving physical function and health-related quality of life in knee and hip OA. ${ }^{3-8}$ In addition, sedentary pursuits have been linked to a decline in physical function irrespective of the time the patients spent in moderate-to-vigorous activities. ${ }^{9}$ Maintaining a physically active lifestyle (ie, time spent in leisure and non-leisure physical activities, not limited to engagement in exercise) is therefore important for people living with lower limb OA. ${ }^{1}$ Nonetheless, the majority of people with knee or hip OA do not meet the guideline recommendations of at least $150 \mathrm{~min}$ of moderate-to-vigorous physical activity per week and are reported to be less 
physically active than their counterparts without OA. ${ }^{10} 11$ Furthermore, in the case of existing exercise interventions in this population, PA maintenance postintervention is a major issue. ${ }^{12} 13$

An emerging question is therefore what are the PA determinants in people with hip or knee OA, so that they can be optimally applied in healthcare practice and policymaking to improve health outcomes. Existing narrative $^{1415}$ and systematic ${ }^{1617}$ reviews have addressed this question. In the most up-to-date quantitative systematic review of factors influencing PA in this population, ${ }^{16}$ demographic characteristics, physical function and symptom severity were the only PA correlates consistently reported by the studies. There was inconsistent association with psychological factors like mental health. The paucity of studies on social and environmental correlates of PA was highlighted in this review. ${ }^{16}$ When it comes to understanding behaviour and behaviour change though, personal (eg, cognitions, attitudes), as well as social and environmental factors are of major importance. ${ }^{18-21}$

To date, no systematic work has captured these factors, with those identified which are modifiable potentially contributing to the development of interventions to promote the initiation and maintenance of $\mathrm{PA}$ in people with OA. Qualitative studies, which offer an in-depth exploration of the human experience, might prove more appropriate in illustrating the variety and interplay of psychosocial and environmental factors that facilitate or hinder PA specifically in people living with lower limb OA. A recent scoping review of quantitative and qualitative studies ${ }^{22}$ has mapped modifiable factors linked to exercise participation in patients with hip and knee OA using the Theoretical Domains Framework. This systematic review of qualitative evidence will move one step further by applying rigorous methodology, such as quality appraisal of the included studies and confidence in the reported findings. Confidence in the reported findings is directly relevant to how informative and useful they can be in practice. In addition, two important distinctions of potential relevance to barriers and facilitators to PA will also be addressed in this systematic review. The first is a discrimination between barriers and facilitators to exercise and 'lifestyle' PA. The second is about the theoretical and empirical distinction between uptake and maintenance of PA, that is, whether PA is a newly introduced or reintroduced behaviour in a person's life or its regular engagement is part of one's lifestyle. ${ }^{23}$ Different factors can act as barriers and facilitators at different stages of behavioural change (in particular, when the focus is on adoption or maintenance), which holds practical implications when it comes to identifying key elements of behavioural interventions.

\section{Objectives}

To identify, appraise and synthesise the existing qualitative evidence on barriers and facilitators to PA uptake and/or maintenance in people with hip or knee OA based on the patients' perceptions and experiences.
Secondary objectives are to explore differences in barriers and facilitators between (1) exercise and lifestyle PA and (2) uptake and maintenance of PA.

\section{METHODS}

This systematic review protocol follows the Preferred Reporting Items for Systematic Review and Meta-analysis Protocols (PRISMA-P) 2015 statement (see online supplementary appendix 1). ${ }^{24} 25$ The systematic review was registered with the International Prospective Register of Systematic Reviews (PROSPERO), registration number CRD42016030024. It will be informed by the Centre for Reviews and Dissemination ${ }^{26}$ and Cochrane Qualitative Research Methods Group ${ }^{27}{ }^{28}$ guidelines and will follow the Enhancing Transparency in Reporting the Synthesis of Qualitative Research (ENTREQ) ${ }^{29}$ and the PRISMA $^{30}$ statements for reporting systematic reviews (see online supplementary appendix 2). In the case of sections applicable to qualitative systematic reviews that are included in PRISMA, but are not covered by ENTREQ, these will also be reported.

\section{Eligibility criteria}

The criteria outlined below will be used for study selection (see online supplementary appendix 3). PICOS (Population, Intervention, Comparison, Outcomes, Study design), which is an established tool for defining key components of research questions, ${ }^{31}$ was adapted for use in this study. In particular, interventions and comparators were not applicable and the phenomenon of interest will be identified instead.

Population: Study participants will be adults who have physician-diagnosed hip or knee OA; or radiographic OA using Kellgren and Lawrence grade $\geq 2$ at hip or knee; or meet internationally accepted classification criteria for OA (eg, American College of Rheumatology classification criteria). If the study population involves groups of patients with other types of arthritis, for example, rheumatoid arthritis, they will be included in this study provided that patients with knee and hip OA combined are the highest proportion of participants. Studies will be excluded if the study participants are people about to undergo or have undergone total hip or knee arthroplasty.

Outcomes will be barriers and facilitators that influence uptake and/or maintenance of PA in people with OA as perceived and reported by the patients.

Studies will be included if (1) they directly explore the factors/barriers/facilitators/motivation that correspond to engagement in PA/exercise (ie, this is stated in the study objectives or relevant interview questions are included); or (2) they directly address or focus on any aspect of the experience or perceptions of people living with hip or knee OA regarding PA and/or exercise.

Study designs: (1) Qualitative studies using appropriate methods of data collection and data analysis. (2) Mixed methods studies that report qualitative findings. 
Language studies will be excluded if written in a language other than English.

Publication year: From database inception to 31 December 2015.

\section{Information sources}

The databases MEDLINE (Ovid MEDLINE(R) In-Process and Other Non-Indexed Citations and Ovid MEDLINE(R) 1946 to Present, OVID interface), EMBASE (1974 onwards, OVID interface), PhychINFO (1967 onwards, OVID interface), Web of Science, CINAHL, SPORTDiscus and Scopus will be searched from inception to 31 December 2015. Also, grey literature sources will be considered, that is, OpenGrey, National Health Service (NHS) evidence. Hand search of qualitative research-centred journals, for example, Qualitative Health Research, Sociology of Health and Illness, will complement the search strategy. Screening of the references of included articles and relevant existing reviews will take place. Finally, active researchers in the field who have contributed to this literature will be contacted.

\section{Search}

The search strategy will comprise comprehensive keyword combinations for each of the four concepts of interest (see online supplementary appendix 4 for MEDLINE), that is, (1) knee and hip OA (1-9 in the online supplementary appendix), (2) PA/exercise (1016), (3) barriers, facilitators, motivation, uptake, maintenance (17-24), (4) qualitative study design (25-30). Free-text search (.mp) will be applied for the basic search terms for each concept (eg, 'osteoarthritis' for population; 'physical activity', 'exercise' for phenomenon of interest; 'barrier*', 'facilitator*', 'motivation' for outcomes; 'qualitative' for study design), supplemented by a wide array of alternative terms searched for in the title/abstract section or free-text search. Within each group of concepts, the keyword combinations will be mutually inclusive ('OR' operator). The combination of the four groups was applied in the latter stage using the AND operator.

\section{Study records}

The study selection process will be according to the PRISMA flow diagram ${ }^{30}$ (see online supplementary appendix 5). Two independent reviewers will run the search and study selection. Endnote X7 software will be used for data management. Citations including abstracts will be imported and duplicates will be removed. Selected articles will be juxtaposed for multiple reports of a single study, so that double counting of studies is avoided.

The predetermined eligibility criteria will be used in the form of a list (see online supplementary appendix 3 ), which will be checked and fine-tuned if necessary by the two reviewers. The reviewers will independently apply the criteria at all stages of the selection process.
After title/abstract screening, full-text copies of potentially relevant studies will be obtained. Additional information will be sought from authors if necessary at the stage of full-text assessment. Where the information provided is insufficient for study selection, assessment and synthesis, the respective studies will not be included in the synthesis but will be referenced in the discussion section. Consensus will be reached through discussion and where agreement is not reached, a third reviewer will be consulted. At the end of the selection process, the $\kappa$ statistic $^{32}$ will be used to assess the chancecorrected agreement between the reviewers in assessing the full-text articles as included, excluded or unclear. A supplementary table with information about the selected studies will be provided including study characteristics (first author's name, publication year, method of data collection and data analysis), participant characteristics (age, gender, locus and severity of OA, duration of diagnosis, physical activity profile) and contextual information (country, geographic area, setting if applicable). Data will be entered in and managed with NVivo V.11 qualitative data analysis software (QSR International).

\section{Data items}

All text under the sections of 'results' and 'findings' will be considered as data and will be analysed. If findings and discussion are presented together, then discussion will also be considered as a data item.

\section{OUTCOMES AND PRIORITISATION}

\section{Phenomenon of interest}

The description and interpretation of patients with OA experiences and perceptions regarding what hinders and what facilitates and motivates them to engage in PA behaviours constitute the phenomenon of interest. All types of factors reported by the participants will be included, for example, health-related, psychological, social, cultural, environmental. Subgroups of the phenomenon of interest will also be explored, provided that there is sufficient evidence. These are: barriers and facilitators to PA uptake and PA maintenance; engagement in exercise and engagement in lifestyle PA.

\section{Appraisal of study quality}

Since there is no consensus on how to assess qualitative evidence and a single set of criteria might not be applicable to all kinds of qualitative research, ${ }^{33}$ two different approaches to appraisal will be applied (see online supplementary appendix 6 ).

First the Critical Appraisal Skills Programme (CASP) Qualitative Checklist, a structured tool commonly employed in systematic reviews (SRs) of qualitative evidence, will be used. CASP Qualitative Checklist is broadly suitable for various qualitative study designs, is available online and free of charge. The tool, including introduction, 10 questions and prompts, will be used as provided by the CASP-uk.net. Studies will be rated as 
'high quality' if they meet at least 8 of the 10 criteria, 'medium quality' if they meet 5-7 of the criteria and 'low quality' if they meet 4 or less.

Although the CASP tool appraises reporting and methodological quality, it does not address aspects of the research validity ${ }^{35}$ and can favour papers that are less insightful as long as they comply with 'expectations of research practice'. ${ }^{36}$ To address this gap, the evaluative criteria of credibility, transferability, dependability and confirmability ${ }^{37}$ will be applied. These criteria widely acknowledge the philosophical stance of qualitative research, focus on the trustworthiness of the study $^{37} 38$ and their development was not aimed in particular at the evaluation of interpretive qualitative approaches as other theoretically informed tools, for example, Popay et al. ${ }^{39}$ Included studies will be assessed as to whether they apply the techniques suggested for ensuring study quality according to Lincoln and Guba's criteria: ${ }^{33} 40$ prolonged engagement, persistent observation, peer review, triangulation, negative case analysis, referential adequacy and member checking to ensure credibility; thick description for transferability; inquiry audit for dependability; confirmability audit, audit trail, triangulation and reflexivity to ensure confirmability. A more detailed description of the context of the above procedures can be found in online supplementary appendix 6. Studies will be rated as 'high quality' if they meet at least three of the four criteria, 'medium quality' if they meet two of the criteria and 'low quality' if they meet one or none.

Two reviewers, both with qualitative research training and experience $(\mathrm{AMK} / \mathrm{NE})$ and one with additional experience in qualitative systematic reviews (NE), will independently appraise the selected studies. First, the appraisal process will be piloted, that is, the reviewers will independently apply the two sets of criteria on two studies and criteria and then compare the outcome and discuss the process they followed, so that potential discrepancies in applying the criteria are resolved. The final assessment for each study will be reached through discussion and in case a consensus is not reached, a third researcher will be consulted. A detailed justification of the assessment outcome for the second set of criteria will be available on publication of the SR.

\section{Data synthesis}

Thematic synthesis as described by Thomas and Harden $^{41}$ will be applied for data synthesis. Thematic synthesis is a transparent and suitable method for integrating qualitative evidence in a SR and has been used for SRs of barriers and facilitators to various behaviours. ${ }^{42-44}$ The synthesis involves three stages: (1) free line-by-line coding; (2) grouping of the codes into 'descriptive themes', which also includes the translation of conceptions from one study to the other (ie, the codes from all included studies will be compared with each other in an iterative process, the codes/quotes describing the same concept will be merged under one code and those expressing a similar concept will be grouped together); and (3) the formation of analytical themes. At the latter stage, barriers and facilitators to PA in people with hip and knee OA will be inferred from the descriptive themes; that is, the research questions, which are put aside during the data-driven first two stages, will be introduced at this point to inform the formation of analytical themes. Therefore, the synthesis will combine both an inductive (at first stages) and a deductive (latter stage) approach. The analytical themes and their relation with descriptive themes will be presented in tables. The synthesis will be conducted by one researcher (AMK) and checked by a second independent reviewer with experience in thematic analysis (NE), to enhance credibility.

\section{Confidence in the synthesised qualitative findings}

Assessing the quality of the studies in a SR does not answer the question of how much certainty or trust we can place on each individual review finding. To ensure the potential value of the review in informing its users, the assessment of the trust that can be placed on each individual finding is advised. ${ }^{45}$ In qualitative evidence syntheses, approaches to confidence in the findings have only recently been developed. ${ }^{38}{ }^{46}$ The ConQual approach, ${ }^{38}$ which was developed by qualitative research experts from the Joanna Briggs Institute in Adelaide, will be adopted for assessing the confidence in the findings. ConQual assesses the confidence in findings, that is, truth value, based on two elements: dependability and credibility (see online supplementary appendix 7). ConQual is the approach of choice as it offers a clear operationalisation of each element and description of the appraisal process. A Confidence in the Findings Table will be formulated which will include the review finding, the assessments for dependability, credibility and the overall confidence score (high, moderate, low, very low).

\section{DISCUSSION}

This systematic review will be the first to synthesise and report barriers and facilitators of PA in people with hip or knee OA based on qualitative evidence. Following the emerging evidence on the independent role of sedentary pursuits on health and mortality ${ }^{47}$ and the shifting of health guidelines and policies from exercise promotion to physical activity promotion, we will further explore differences between determinants of lifestyle PA and exercise, as there is a pronounced gap in the literature regarding the former. ${ }^{49}$ Additionally, we will explore differences reported in the literature between uptake and maintenance of PA. The review findings will inform our understanding of factors facilitating or inhibiting participation in physical activity and provide information on how to optimise behaviour change at different stages (ie, uptake or maintenance) in the targeted population. 
This protocol serves to provide a detailed account of the rational and methods to be used in the proposed systematic review to ensure the transparency of the process. ${ }^{24}$ In case any deviation from the protocol takes place, it will be justified and discussed in the systematic review on publication.

Acknowledgements Dr Nikolaos Efstathiou provided feedback on the choice of tools for quality appraisal and data synthesis.

Collaborators Dr Nikolaos Efstathiou.

Contributors JLD, AMK, AR, RK and AA contributed to the development of the study design and search strategy. RK and AA provided expertise on the selection criteria. AR provided expertise on the methodological issues related to systematic reviews. AMK developed the SR protocol and all authors provided feedback and approved the final protocol.

Funding This review will comprise part of the research requirements of a $\mathrm{PhD}$ to be completed by AMK, which has received funding by the MRC-Arthritis Research UK Centre for Musculoskeletal Ageing Research.

Competing interests None declared.

Provenance and peer review Not commissioned; externally peer reviewed.

Open Access This is an Open Access article distributed in accordance with the terms of the Creative Commons Attribution (CC BY 4.0) license, which permits others to distribute, remix, adapt and build upon this work, for commercial use, provided the original work is properly cited. See: http:// creativecommons.org/licenses/by/4.0/

\section{REFERENCES}

1. NICE. Osteoarthritis care and management in adults. NICE clinical guideline, 2014

2. Caspersen CJ, Powell KE, Christenson GM. Physical activity, exercise, and physical fitness: definitions and distinctions for health-related research. Public Health Rep 1985;100:126-31.

3. Anwer S, Alghadir A, Brismée JM. Effect of home exercise program in patients with knee osteoarthritis: a systematic review and meta-analysis. J Geriatr Phys Ther 2016;39:38-48.

4. Juhl C, Christensen R, Roos EM, et al. Impact of exercise type and dose on pain and disability in knee osteoarthritis: a systematic review and meta-regression analysis of randomized controlled trials. Arthritis Rheumatol 2014;66:622-36.

5. Fransen M, McConnell S, Hernandez-Molina G, et al. Exercise for osteoarthritis of the hip. Cochrane Database Syst Rev 2014(4): CD007912.

6. Fransen M, McConnell S, Harmer AR, et al. Exercise for osteoarthritis of the knee: a Cochrane systematic review. $\mathrm{Br} J$ Sports Med 2015;49:1554-7.

7. Tanaka R, Ozawa J, Kito $\mathrm{N}$, et al. Does exercise therapy improve the health-related quality of life of people with knee osteoarthritis? A systematic review and meta-analysis of randomized controlled trials. $J$ Phys Ther Sci 2015;27:3309-14.

8. Uthman OA, van der Windt DA, Jordan JL, et al. Exercise for lower limb osteoarthritis: systematic review incorporating trial sequential analysis and network meta-analysis. Br J Sports Med 2014;48:1579.

9. Semanik PA, Lee J, Chang RW, et al. Accelerometer-monitored sedentary behavior and observed physical function loss. Am J Public Health 2015;105:560-6.

10. Wallis JA, Webster KE, Levinger $\mathrm{P}$, et al. What proportion of people with hip and knee osteoarthritis meet physical activity guidelines? A systematic review and meta-analysis. Osteoarthritis Cartilage 2013;21:1648-59.

11. Herbolsheimer F, Schaap LA, Edwards MH, et al., Eposa Study Group. Physical activity patterns among older adults with and without knee osteoarthritis in six European countries. Arthritis Care Res (Hoboken) 2016;68:228-36.

12. Pisters MF, Veenhof C, van Meeteren NL, et al. Long-term effectiveness of exercise therapy in patients with osteoarthritis of the hip or knee: a systematic review. Arthritis Rheum 2007;57:1245-53.

13. Williamson W, Kluzek S, Roberts N, et al. Behavioural physical activity interventions in participants with lower-limb osteoarthritis: a systematic review with meta-analysis. BMJ Open 2015;5:e007642.
14. Marks R. Knee osteoarthritis and exercise adherence: a review. Curr Aging Sci 2012;5:72-83.

15. Marks R, Allegrante JP. Chronic osteoarthritis and adherence to exercise: a review of the literature. J Aging Phys Act 2005;13:434-60.

16. Stubbs B, Hurley M, Smith $T$. What are the factors that influence physical activity participation in adults with knee and hip osteoarthritis? A systematic review of physical activity correlates. Clin Rehabil 2015;29:80-94.

17. Veenhof C, Huisman PA, Barten JA, et al. Factors associated with physical activity in patients with osteoarthritis of the hip or knee: a systematic review. Osteoarthritis Cartilage 2012;20:6-12.

18. Ajzen I. The theory of planned behavior. Organ Behav Hum Decis Processes 1991;2:179-211.

19. Bandura A. Health promotion from the perspective of social cognitive theory. Amsterdam, The Netherlands: Harwood Academic Publishers, 2000

20. Deci EL, Ryan RM. Self-determination theory: a macrotheory of human motivation, development, and health. Can Psychol 2008;49:182-5

21. Sallis J, Owen N, Fisher EB. Health behavior and health education: theory, research and practice. 4th edn. San Fransisco, CA, USA: Jossey-Bass, 2008

22. Dobson F, Bennell KL, French SD, et al. Barriers and facilitators to exercise participation in people with hip and/or knee osteoarthritis: synthesis of the literature using behavior change theory. Am J Phys Med Rehabil 2016:95:372-89.

23. Prochaska JO, Di Clemente CC. Transtheoretical therapy: toward a more integrative model of change. Psychotherapy 1982;19:276-88.

24. Moher D, Shamseer L, Clarke M, et al. Preferred reporting items for systematic review and meta-analysis protocols (PRISMA-P) 2015 statement. Syst Rev 2015;4:1.

25. Shamseer L, Moher D, Clarke M, et al. Preferred reporting items for systematic review and meta-analysis protocols (PRISMA-P) 2015: elaboration and explanation. BMJ 2015;349:g7647.

26. CRD. Systematic reviews. CRD's guidance for undertaking reviews in health care. University of York: Centre for Reviews and Dissemination, 2009.

27. Noyes J, Popay J, Pearson A, et al. Qualitative research and Cochrane reviews. Cochrane handbook for systematic reviews of interventions. John Wiley \& Sons, Ltd, 2008:571-91.

28. Noyes J, Lewin S. Chapter 6: Supplemental Guidance on Selecting a Method of Qualitative Evidence Synthesis, and Integrating Qualitative Evidence with Cochrane Intervention Reviews. In: Noyes J, Booth A, Hannes K, et al, eds. Supplementary Guidance for Inclusion of Qualitative Research in Cochrane Systematic Reviews of Interventions. Version 1 (updated August 2011) Cochrane Collaboration Qualitative Methods Group, 2011:3-10. http://cqrmg.cochrane.org/supplemental-handbook-guidance

29. Tong A, Flemming K, Mclnnes $\mathrm{E}$, et al. Enhancing transparency in reporting the synthesis of qualitative research: ENTREQ. BMC Med Res Methodol 2012;12:181.

30. Moher D, Liberati A, Tetzlaff J, et al. Preferred reporting items for systematic reviews and meta-analyses: the PRISMA statement. Ann Intern Med 2009;151:264-9. w64.

31. O'Connor D, Green S, Higgins JPT. Defining the review question and developing criteria for including studies. Cochrane handbook for systematic reviews of interventions. John Wiley \& Sons, Ltd, 2008:81-94

32. McHugh ML. Interrater reliability: the kappa statistic. Biochem Med (Zagreb) 2012;22:276-82

33. Creswell JW. Qualitative inquiry and research design: choosing among five approaches. Thousand Oaks: Sage Publications, Inc, 2007.

34. Sparkes A, Smith B. Qualitative research methods in sport, exercise and health. New York: Routledge, 2014

35. Critical Appraisal Skills Programme (CASP). CASP Checklists (http:/ wwwcasp-uknet/\#!checklists/cb36). Oxford: CASP, 2014.

36. Dixon-Woods M, Sutton A, Shaw R, et al. Appraising qualitative research for inclusion in systematic reviews: a quantitative and qualitative comparison of three methods. J Health Serv Res Policy 2007;12:42-7.

37. Lincoln Y, Guba E. Naturalistic inquiry. Newbury Park, CA: Sage Publications, 1985.

38. Munn Z, Porritt K, Lockwood C, et al. Establishing confidence in the output of qualitative research synthesis: the ConQual approach. BMC Med Res Methodol 2014;14:1-7.

39. Popay J, Rogers A, Williams G. Rationale and standards for the systematic review of qualitative literature in health services research. Qual Health Res 1998;8:341-51.

40. Cohen D, Crabtree B. Qualitative Research Guidelines Project. http:// www.qualres.org/HomePopa-3686.html (accessed 21 Jan 2016), July 2006 
41. Thomas J, Harden A. Methods for the thematic synthesis of qualitative research in systematic reviews. BMC Med Res Methodol 2008;8:45.

42. Anderson K, Stowasser D, Freeman C, et al. Prescriber barriers and enablers to minimising potentially inappropriate medications in adults: a systematic review and thematic synthesis. BMJ Open 2014;4: e006544.

43. Ferrer HB, Trotter C, Hickman M, et al. Barriers and facilitators to HPV vaccination of young women in high-income countries: a qualitative systematic review and evidence synthesis. BMC Public Health 2014;14:700.

44. Mazarello Paes V, Ong KK, Lakshman R. Factors influencing obesogenic dietary intake in young children (0-6 years): systematic review of qualitative evidence. BMJ Open 2015;5:e007396.

45. Lewin S, Bosch-Capblanch X, Oliver S, et al. Guidance for evidence-informed policies about health systems: assessing how much confidence to place in the research evidence. PLoS Med 2012;9:e1001187.

46. Lewin $\mathrm{S}$, Glenton $\mathrm{C}$, Munthe-Kaas $\mathrm{H}$, et al. Using qualitative evidence in decision making for health and social interventions: an approach to assess confidence in findings from qualitative evidence syntheses (GRADE-CERQual). PLoS Med 2015;12: e1001895

47. Loprinzi PD, Loenneke JP, Ahmed HM, et al. Joint effects of objectively-measured sedentary time and physical activity on all-cause mortality. Prev Med 2016;90:47-51.

48. de Rezende LF, Rodrigues Lopes M, Rey-López JP, et al. Sedentary behavior and health outcomes: an overview of systematic reviews. PLOS ONE 2014:9:e105620.

49. Koeneman MA, Verheijden MW, Chinapaw MJ, et al. Determinants of physical activity and exercise in healthy older adults: a systematic review. Int J Behav Nutr Phys Act 2011;8:142. 